\section{Discussion}

Although SAE rarely occurs, it is a recognized complication of thoracic trauma, including iatrogenic injuries. Intraoperative SAE after stapled wedge excision of a pulmonary nodule has been reported; transesophageal echocardiography identified air in the left atrium, but the patient had no neurologic sequelae. ${ }^{2}$

CAE has been reported after CT-guided needle localization ${ }^{3}$ and bronchoscopy with insufflation of oxygen at high pressure, ${ }^{4}$ but it has not been reported after a pulmonary resection. Neurologic sequelae are noted immediately after CAE. ${ }^{3-5}$ Our patient had no neurologic symptoms until 6 hours after extubation and 4 hours after commencement of NIPPV. We believe that air entered the circulation because of the increased airway pressure generated by BIPAP rather than intraoperatively. CAE has been reported after 2 weeks of BIPAP in a patient with post-bone marrow transplant pneumonitis. ${ }^{5}$

SAE requires the presence of a portal of entry and a pressure gradient to drive the gas into the circulation. In a spontaneously breathing patient, the pressure in the airways is lower than in the pulmonary veins. With the institution of positive pressure ventilation, the gradient alters in favor of entrainment of air into the pulmonary venous system. Whether the air was entrained through a fistulous communication at the lung periphery because of the surgery or a new communication secondary to NIPPV in an emphysematous lung is unknown. Both peripheral ${ }^{2,3}$ and centrally ${ }^{4}$ located pulmonary interventions have been implicated in air embolism.

The diagnosis of cerebral air embolus is often clinical; head CT is frequently normal ${ }^{3,4}$ or only shows areas of embolic infarc- tion. ${ }^{1,5}$ Intracranial air is rarely visualized. Hyperbaric oxygen therapy has been used to treat patients with $\mathrm{CAE},{ }^{4}$ but it is seldom used $^{1,3}$ because of the difficulties in making the diagnosis with certainty, transporting a critically ill patient, and accessing facilities that provide hyperbaric oxygen. With purely supportive patient treatment, CAE usually causes transient neurologic sequelae with complete recovery within 2 weeks ${ }^{1,3}$; when patients have died, this was attributed to other causes. ${ }^{5}$

We report a case of CAE after pulmonary wedge excision, of which we are not aware of any other reports. It is unusual to obtain radiologic evidence of the diagnosis, and its precipitation by noninvasive ventilation has not been reported after pulmonary surgery.

\section{References}

1. Brownlow HA, Edibam C. Systemic air embolism after intercostal chest drain insertion and positive pressure ventilation in chest trauma. Anaesth Intensive Care. 2002;30:660-4.

2. Hemmerling TM, Schmidt J, Bosert C, et al. Systemic air embolism during wedge resection of the lung. Anesth Analg. 2001;93:1135-6.

3. Kamiyoshihara M, Sakata K, Ishikawa $\mathrm{S}$, et al. Cerebral arterial air embolism following CT-guided lung needle marking. J Cardiovasc Surg. 2001;42:699-700.

4. Wherrett CG, Mehran RJ, Beaulieu MA. Cerebral arterial gas embolism following diagnostic bronchoscopy: delayed treatment with hyperbaric oxygen. Can J Anesth. 2002;49:96-9.

5. Hung S-C, Hsu H-C, Chang S-C. Cerebral air embolism complicating bilevel positive airway pressure therapy. Eur Respir J. 1998;12:235-7.

\title{
Concurrent primary cardiac tumors
}

\author{
Tulsi Menon, MBBS, Yuki Watanabe, MBBS, and David Andrews, MBBS, FRACS, Perth, Australia
}

\section{Clinical Summary}

An 80-year-old woman was referred to a cardiologist with recurrent palpitations, atrial fibrillation, and flutter. Investigation with an echocardiogram revealed a mass (believed to be a myxoma) on the fossa ovalis, extending into the left atrium and then out near the right superior pulmonary vein, where it became frond-like and friable. She was subsequently referred for surgical intervention to remove the myxoma. She was otherwise relatively well.

From the Department of Cardiothoracic Surgery, Royal Perth Hospital, Perth, Western Australia, Australia.

Received for publication Feb 2, 2007; accepted for publication Feb 12, 2007.

Address for reprints: Tulsi Menon, MBBS, 19 Morgan Rd, Redcliffe WA 6104, Australia (E-mail: kaltul@optusnet.com.au).

J Thorac Cardiovasc Surg 2007;134:263-4

0022-5223/\$32.00

Copyright $\odot 2007$ by The American Association for Thoracic Surgery

doi:10.1016/j.jtcvs.2007.02.038
Intraoperatively, after the patient was commenced on bypass and the aorta was crossclamped, an incision to the atrial septum was performed on the tricuspid valve side of the fossa ovalis. Affixed to the atrial fossa was a sessile mass, the atrial myxoma, extending into the left atrium. Distant from this mass, situated in the posterior wall of the left atrium (near the origin of the right superior pulmonary vein), was a frond-like mass, a papillary fibroelastoma, with frond lengths measuring around $1.5 \mathrm{~cm}$ and the base densely filled with tentacles. Both tumors were excised, and the defects were closed. The patient was taken off bypass. Closure was performed in the routine manner.

The patient was transferred to the intensive care unit postoperatively, discharged to the ward 3 days later, and discharged home 11 days postoperatively. Histopathology of the resected left atrial lesions confirmed atrial myxoma and papillary fibroelastoma. Follow-up echocardiograms several months and more than 1 year postoperatively demonstrated no evidence of remaining tumor.

\section{Discussion}

Primary cardiac tumors are very uncommon, with myxomas as the most common benign primary cardiac tumor. Myxomas commonly 
occur in persons aged 30 to 60 years, although all ages can be affected. Up to $80 \%$ of myxomas are contained in the left atrium, and $7 \%$ to $20 \%$ are contained in the right atrium. The remaining are found either biatrially or in the right or left ventricle. ${ }^{1}$ Although benign, myxomas can be fatal because of embolic complications. However, many are asymptomatic, with myxomas discovered incidentally on echocardiogram. Treatment of myxomas in adults involves surgical resection. ${ }^{1}$

Cardiac papillary fibroelastomas are the second most common primary cardiac tumor in adults. ${ }^{2}$ Papillary fibroelastomas occur in all age groups, with a mean age of around 60 years. They can develop anywhere in the heart but predominantly on the cardiac valvular endocardium. The majority of these tumors arise from the left side of the heart. Characteristically, they have a flower-like appearance, with multiple papillary fronds attached to the endocardium by a central core (like a sea anemone). Symptoms are usually associated with embolization. It has been reported that around $30 \%$ of patients are asymptomatic, receiving diagnoses incidentally either by means of echocardiography, intraoperatively, or at autopsy. ${ }^{2}$ Patients with sizeable $(>1 \mathrm{~cm})$ and mobile tumors or who have had embolic occurrences require surgical intervention. $^{2}$

Primary cardiac tumors are an uncommon finding; the discovery of different primary cardiac tumors located concurrently is particularly rare. Review of the literature has yielded, to our knowledge, 3 cases in the past in which more than one type of primary cardiac tumor has been discovered concomitantly.

A papillary fibroelastoma of an aortic valve coexistent with a cystic tumor of the atrioventricular valve found during autopsy was reported by Agaimy and Mandi. ${ }^{3}$ Prifti and associates ${ }^{4}$ reported a case of a myxoma associated with a papillary fibroelastoma. Both these tumors were situated on the anterior mitral valve, with the myxoma derived from the atrial surface and the papillary fibroelastoma from the ventricular surface of the valve. Akiyama and coworkers ${ }^{5}$ recounted a case of a symptomatic left atrial (involving the atrial septum) cardiac myxoma coexistent with two papillary fibroelastomas on the aortic valve.

In all three cases, the papillary fibroelastoma has been found arising from either the mitral valve or the aortic valve. We report a case of both the myxoma and papillary fibroelastoma found attached to the left atrial septum (mainly the posterior wall of the left atrium), which to our knowledge has not been reported in the literature before. In this patient, what was initially thought to be one tumor (ie, a myxoma extending into the left atrium and then out near the right superior pulmonary vein, where it became frond-like and friable) was actually two different types of tumors. The mass on the fossa ovalis extending into the left atrium was the myxoma, with the papillary fibroelastoma being the frond-like and friable part near the right superior pulmonary vein.

In summary, although the simultaneous existence of two different primary cardiac tumors is rare, it is a possible occurrence, and hence when evaluating a patient preoperatively with a primary cardiac tumor (or intraoperatively when removing a tumor), the potential for an additional tumor or tumors occurring concurrently must be considered.

\section{References}

1. Keeling IM, Oberwalder P, Anelli-Monti M, Schuchlenz H, Demel U, Tilz GP, et al. Cardiac myxomas: 24 years of experience in 49 patients. Eur J Cardiothorac Surg. 2002;22:971-7.

2. Gowda RM, Khan IA, Nair CK, Mehta NJ, Vasavada BC, Sacchi TJ. Cardiac papillary fibroelastoma; a comprehensive analysis of 725 cases. Am Heart J. 2003;146:404-10.

3. Agaimy A, Mandi L. Papillary fibroelastoma of the aortic valve coexisting with a cystic tumor of the atrioventricular nodal region: a case report. Pathologe. 2000;21:250-4.

4. Prifti E, Bonacchi M, Salica A. Mitral valve myxoma concomitant with papillary fibroelastoma. Ann Thorac Surg. 2000;70: 335-6.

5. Akiyama K, Hirota J, Tsuda Y, Ebishima H, Li C. Double primary cardiac tumors: possible association with a variety of cardiac diseases. J Cardiovasc Surg. 2006;47:81-2. 